\title{
Análise da hidratação do arroz na parboilização
}

\author{
Water absorption kinetics in parboiled husk rice
}

\author{
Fernando Mendes BOTELHO ${ }^{1 \star}$, Paulo César CORREAA ${ }^{1}$, André Luis Duarte GONELI ${ }^{1}$, \\ Marcio Aredes MARTINS ${ }^{1}$, Fernanda Machado BAPTESTINI ${ }^{1}$
}

\section{Resumo}

O objetivo deste trabalho foi estudar o fenômeno de hidratação em grãos de arroz da variedade IRGA 424 para diferentes temperaturas. Foram utilizados grãos de arroz em casca com teor de água inicial de $0,1364 \mathrm{~kg}_{\mathrm{a}} \cdot \mathrm{kg}_{\mathrm{ms}}^{-1}$ embebidos em água destilada nas temperaturas de 35 , 45,55 e $75^{\circ} \mathrm{C}$. O aumento da temperatura resultou em aumento da taxa de absorção de água e o modelo de Peleg ajustou-se satisfatoriamente aos dados experimentais. O coeficiente efetivo de difusão aumentou com o aumento da temperatura variando de 0,80 a $9,18 \times 10^{-11} \mathrm{~m}^{2} / \mathrm{s}$. A dependência do coeficiente efetivo de difusão com a temperatura pode ser descrita pela relação de Arrhenius, apresentando, para a faixa de temperatura estudada, a energia de ativação de $33,2 \mathrm{~kJ} \cdot \mathrm{mol}^{-1}$.

Palavras-chave: hidratação; difusão; Peleg; arroz em casca.

\begin{abstract}
The aim of this work was to study the water uptake phenomenon during soaking of husk rice (IRGA 424) under different temperatures. Grains of husk rice with initial water content of 0.1364 (d.b.) imbibed in distilled water at temperatures of $35,45,55$, and $75^{\circ} \mathrm{C}$ were used. A temperature increase resulted in an increase in the water absorption rate. The Peleg's model fitted satisfactory to the experimental data of water absorption kinetics. The effective diffusion coefficient increased with an increase in temperature ranging from 0.80 to $9.18 \times 10^{-11} \mathrm{~m}^{2} / \mathrm{s}$. The dependency of the effective diffusion coefficient on temperature can be described by the Arrhenius relationship for the temperature range studied, activation energy of $33.2 \mathrm{~kJ} \cdot \mathrm{mol}^{-1}$.
\end{abstract}

Keywords: hydration; diffusion; Peleg; husk rice.

\section{Introdução}

O arroz é uma das principais fontes alimentares da humanidade, sendo que no Brasil, juntamente com o feijão, representa a base da alimentação da sua população. Apesar de ser grande consumidor, o Brasil não é um grande produtor, necessitando importar grande parte do arroz consumido no país, principalmente do Uruguai e da Argentina. O brasileiro tem demonstrado maior exigência no que diz respeito à qualidade deste produto, preferindo arroz tipo 1 ou tipo 2 e o parboilizado. No ano de 2007, a produção brasileira foi de aproximadamente 11 milhões de toneladas, mantendo assim a produção do ano anterior (INSTITUTO..., 2007).

As operações de secagem, adsorção e hidratação do arroz estão presentes em importantes etapas da parboilização desse produto. A parboilização é essencialmente um processo hidrotérmico, no qual o arroz em casca é imerso em água potável a uma dada temperatura para realizar a gelatinização total ou parcial do amido presente nele. $O$ processo que acontece quando o arroz atinge a temperatura de gelatinização, causa uma ruptura na estrutura cristalina dos grânulos de amido contidos no produto, começam então a intumescer e a formar soluções consideravelmente viscosas promovendo um excesso de água no grão.
A parboilização causa modificações químicas e físicas no grão, produzindo mudanças favoráveis à sua utilização, tais como, descascar mais fácil, maior rendimento com menos grãos quebrados, maior resistência a insetos, textura do arroz cozido mais firme, menor lixiviação de sólidos durante o cozimento e maior retenção de nutrientes no grão (como, vitaminas e minerais) (IGATHINATHANE; CHATTOPADHYAY; PORDESIMO, 2005). Estudar a operação de hidratação do arroz parboilizável, torna-se importante para conhecer todas as etapas subsequentes ao seu processamento e a qualidade final do produto.

Muitos estudos envolvendo a cinética de adsorção e absorção em diversos produtos agrícolas como trigo (MASKAN, 2001, 2002; KORNARZYNSKI; PIETRUSZEWSKI; LACEK, 2002), soja (BAYRAM; KAYA; ÖNER, 2004), grão-de-bico (SAYAR; TURHAN; GUNASEKARAN, 2001; TURHAN; SAYAR; GUNASEKARAN, 2002; GOWEN et al., 2007), legumes (SEYHAN-GÜRTAS; MEHMET; EVRANUZ, 2001) ou em produtos industrializados como macarrão penne (CUNNINGHAM; McMINN; RICHARDSON, 2007) e cereais (LUCAS; RAY; MARIETTE, 2007), têm demonstrado o interesse e a sua importância. Nesses estudos, têm-se identificado a influência da temperatura na taxa e na quantidade de água difundida para o interior do produto.

Recebido para publicação em 24/6/2008

Aceito para publicação em 26/6/2009 (003610)

${ }^{1}$ Centro Nacional de Treinamento em Armazenagem, Universidade Federal de Viçosa - UFV, Campus UFV, CEP 36570-000, Viçosa - MG, Brasil,

E-mail:fernando_eaa@yahoo.com.br

${ }^{*}$ A quem a correspondência deve ser enviada 
A modelagem matemática é essencial para predizer e simular o comportamento dos materiais submetidos a determinado processo, podendo-se utilizar os modelos, teóricos e empíricos. Os primeiros, baseados em leis e teorias, são mais complexos e envolvem parâmetros que não são adequados para práticas computacionais na maioria das situações (MASKAN, 2002). Já os modelos empíricos, apesar de normalmente não possuírem uma fundamentação teórica, geralmente são mais simples e de fácil aplicação. O método empírico fundamenta-se na análise de dados experimentais, na análise dimensional e na análise estatística.

Dentre os modelos teóricos aplicados ao processo de hidratação, o modelo da difusão líquida é o mais comumente investigado. Este modelo é aplicado em processos de secagem e de hidratação de um sólido a uma taxa decrescente, considerando como mecanismo principal a difusão líquida baseada na Lei de Fick, que estabelece uma relação entre as taxas de variação temporal e espacial da concentração de água, por meio do coeficiente de difusão. Para que ocorra a difusão de água em grãos é necessário, basicamente, que exista uma força motriz, ou seja, um gradiente de concentração de água entre a superfície e o interior do produto. Todavia, alterações físico-químicas ocorrem em paralelo à difusão de água nos produtos agrícolas, sendo observadas alterações na composição química e na estrutura física desses produtos. Em produtos capilares porosos, como a maioria dos produtos agrícolas, os possíveis mecanismos de transporte de água são: difusão líquida, difusão capilar, difusão na superfície, fluxo hidrodinâmico, difusão de vapor e difusão térmica (BROOKER; BAKKER-ARKEMA; HALL, 1992).

As isotermas de hidratação obtidas experimentalmente, sob condições controladas, fornecem informações sobre o mecanismo de transporte de água e são utilizadas para determinação do coeficiente efetivo de difusão. O coeficiente de difusão determinado experimentalmente é considerado como efetivo ou aparente por que os modelos baseados na segunda Lei de Fick não são rigorosamente representativos dos diversos mecanismos que prevalecem no transporte de água em produtos agrícolas (ROCA et al., 2008).

Muitos pesquisadores têm verificado que a dependência do coeficiente efetivo de difusão com a temperatura pode ser expressa por meio da representação de Arrhenius (RESIO; AGUERRE; SUAREZ, 2003; ADDO; BART-PLANGE; DZISI, 2006; BELLO; TOLABA; SUAREZ, 2007; GELY; GINER, 2007; GELY; SANTALLA, 2007).

Entre os modelos empíricos, um que vem sendo frequentemente utilizado para a representação da hidratação em produtos alimentícios, é o proposto por Peleg (1988), conhecido como modelo de Peleg. A maior vantagem deste modelo é sua simplicidade em relação aos outros modelos teóricos e empíricos. Este modelo já foi utilizado satisfatoriamente para descrever o fenômeno de hidratação em: cogumelos (GARCÎA-PASCUAL et al., 2006), feijão (ABU-GHANNAM; McKENNA, 1997), grão-de-bico (TURHAN; SAYAR; GUNASEKARAN, 2002), dentre outros.

Diante do exposto, com o presente trabalho, objetivou-se modelar para diferentes temperaturas, a operação de hidratação dos grãos de arroz em casca da variedade IRGA 424, bem como verificar a influência deste processo na difusão de água para o interior do sólido.

\section{Material e métodos}

Este trabalho foi realizado no Laboratório de Propriedades Físicas e Avaliação da Qualidade, pertencente ao Centro Nacional de Treinamento em Armazenagem (CENTREINAR) localizado no Campus da Universidade Federal de Viçosa UFV, Viçosa - MG.

\subsection{Absorção de água}

Para realização do experimento, utilizou-se arroz em casca, variedade IRGA 424 proveniente da região de Pelotas - RS, onde, inicialmente, foi feita uma seleção do material a fim de eliminar grãos defeituosos e materiais estranhos. O teor de água inicial médio da amostra foi de $0,1364 \mathrm{~kg}_{\mathrm{a}} \mathrm{kg}_{\mathrm{ms}}^{-1}(\mathrm{~kg}$ de água por $\mathrm{kg}$ matéria seca), sendo este determinado utilizando uma estufa com circulação forçada de ar à temperatura de $105 \pm 1{ }^{\circ} \mathrm{C}$ por 24 horas em três repetições (BRASIL, 1992).

No processo de hidratação foram utilizadas amostras de $100 \mathrm{~g}$ de arroz, que foram embebidas com água destilada em um Becker numa relação de quatro volumes de água para um de produto, às temperaturas de $35,45,55$ e $75^{\circ} \mathrm{C}$, em banho-maria, por um período de dez horas, com três repetições por temperatura. Durante esse período, as amostras foram periodicamente pesadas em uma balança digital com precisão 0,01 g. Para tal, as amostras foram retiradas da imersão e deixadas sobre papel toalha por três minutos, para que a água superficial fosse retirada, pesadas e posteriormente retornavam à imersão.

O teor de água para um dado instante depois do início do experimento foi calculado com base no aumento de massa das amostras em relação à massa inicial.

\subsection{Modelagem da operação de hidratação}

Utilizou-se, para a modelagem da hidratação do arroz, o tradicional modelo de Peleg (1988), apresentado a seguir (Equação 1):

$$
U_{t}=U_{0}+\frac{t}{\left(C_{1}+C_{2} t\right)}
$$

em que: as constantes $C_{1}$ e $C_{2}$ do modelo proposto para modelagem do processo de hidratação dos grãos de milho foram obtidas pelo ajuste da Equação de Peleg linearizada, conforme descrito na Equação 2:

$\frac{t}{U_{t}-U_{0}}=C_{1}+C_{2} t$

em que: $U_{\mathrm{t}}$ teor de água para num dado tempo, $\mathrm{kg}_{\mathrm{a}}\left(100 \mathrm{~kg}_{\mathrm{ms}}\right)^{-1}$; $U_{0}$ : teor de água inicial do produto, $\mathrm{kg}_{\mathrm{a}}\left(100 \mathrm{~kg}_{\mathrm{ms}}\right)^{-1} ; C_{1}$ : taxa constante de Peleg, $\left(100 \mathrm{~h} \mathrm{~kg}_{\mathrm{ms}}\right) \mathrm{kg}_{\mathrm{a}}^{-1} ; C_{2}$ : capacidade constante de Peleg, $\left(100 \mathrm{~kg}_{\mathrm{ms}}\right) \mathrm{kg}_{\mathrm{a}}^{-1}$; e $t$ : tempo, horas.

Uma das grandes vantagens do modelo de Peleg é o ganho de tempo para predizer a cinética de sorção de água em alimentos, possibilitando o cálculo do teor de água de equilíbrio (PELEG, 1988). O teor de água de equilíbrio pode ser obtido pela própria Equação 1, quando o tempo tende ao infinito, resultando em (Equação 3):

$U_{e}=U_{0}+\frac{1}{C_{2}}$ 
em que: $U_{\mathrm{e}}$ : teor de água de equilíbrio, $\mathrm{kg}_{\mathrm{a}}\left(100 \mathrm{~kg}_{\mathrm{ms}}\right)^{-1}$.

\subsection{Determinação do coeficiente efetivo de difusão}

O coeficiente efetivo de difusão foi obtido por meio do ajuste do modelo matemático da difusão líquida, descrito pela Equação 4, aos dados experimentais de hidratação do arroz. Essa equação é a solução analítica para a segunda Lei de Fick, considerando a forma geométrica teórica de um cilindro infinito, desconsiderando a expansão volumétrica dos grãos e considerando a condição de contorno de equilíbrio de umidade conhecida na superfície do grão (BROOKER; BAKKERARKEMA; HALL, 1992).

$R U=\frac{U_{t}-U_{e}}{U_{0}-U_{e}}=\sum_{i=1}^{\infty} \frac{4}{\lambda_{n}{ }^{2}} \exp \left[\frac{-l_{n}{ }^{2} D_{e f} t}{r^{2}}\right]$

em que: $R U$ : razão de umidade, adimensional; $\lambda n$ : raízes da função de Bessel de ordem zero; $D_{\text {ef: }}$ coeficiente efetivo de difusão, $\mathrm{m}^{2} / \mathrm{s}$; e $r$ : raio equivalente, $\mathrm{m}$.

A solução analítica dessa equação apresenta-se na forma de uma série infinita de rápida convergência. Desta forma, um número finito de termos $(n)$ pode ser utilizado para uma precisão especificada.

$\mathrm{O}$ raio equivalente de um grão pode ser definido como o raio da esfera com mesmo volume. Para se determinar o volume médio dos grãos de arroz, considerou-se o arroz como sendo um cilindro reto. Para cálculo do seu volume, tomou-se uma amostra de cinquenta grãos, na qual foram realizadas medidas no comprimento, largura e espessura, com a ajuda de um paquímetro digital com precisão de $0,01 \mathrm{~mm}$, sendo que o diâmetro do cilindro foi considerado a média das medidas da largura e espessura. $\mathrm{O}$ raio equivalente encontrado neste trabalho foi de $0,00265 \mathrm{~m}$.

\subsection{Efeito da temperatura}

A dependência do coeficiente efetivo de difusão com a temperatura foi verificada utilizando-se a relação Arrhenius, Equação 5:

$$
D_{e f}=D_{0} \exp \left(\frac{-E_{a}}{R T_{a}}\right)
$$

Em que: $E_{\mathrm{a}}$ : energia de ativação, $\mathrm{kJ} \cdot \mathrm{mol}^{-1} ; R$ : constante universal dos gases, $\mathrm{kJ} \mathrm{mol}^{-1} \cdot \mathrm{K}^{-1} ; T_{\mathrm{a}}$ : temperatura absoluta, $\mathrm{K}$; e $D_{0}$ : fator pré-exponencial equivalente ao coeficiente de difusão para temperaturas tendendo ao infinito, $\mathrm{m}^{2} / \mathrm{s}$.

\subsection{Análise estatística}

Para verificar o grau de ajuste do modelo, considerou-se a significância dos coeficientes do modelo, as magnitudes do coeficiente de determinação $\left(\mathrm{R}^{2}\right)$, do erro médio relativo $(P)$ e da estimativa do desvio padrão $(S E)$. Os parâmetros $P$ e $S E$ foram calculados conforme as Equações 6 e 7, respectivamente:

$$
P=\frac{100}{n} \sum \frac{|Y-\hat{Y}|}{Y}
$$

$S E=\sqrt{\frac{\sum(Y-\hat{Y})^{2}}{G L R}}$

em que: $Y$ : valor observado experimentalmente; $\hat{Y}$ : valor estimado pelo modelo; $n$ : número de dados observados; e GLR: graus de liberdade do modelo (número de dados observados menos o número de parâmetros do modelo).

\section{Resultados e discussão}

\subsection{Isotermas de absorção água}

Na Figura 1 são apresentadas as variações do teor de água do arroz em casca para as temperaturas estudadas em relação ao tempo.

Nota-se, pela Figura 1, que o arroz exibiu um comportamento característico de produtos agrícolas submetidos ao processo de hidratação. Observa-se que o início do processo de hidratação exibe uma alta taxa de absorção de água, tendendo a diminuir e estabilizar com o tempo, à medida que o teor de água do produto se aproxima do equilíbrio. Bello, Tolaba e Suarez (2004) argumentam que este fato pode ser atribuído à inibição capilar das camadas externas do pericarpo que apressaria a absorção de água. Para o caso do arroz, essa absorção também é afetada pela presença ou não da casca. Outro fator ao qual poderiam ser atribuídas as altas taxas iniciais de absorção de água é o alto potencial matricial dos vários tecidos constituintes do grão.

Nota-se também um aumento regular na absorção de água na medida em que se aumenta a temperatura até $55^{\circ} \mathrm{C}$. Entretanto, um comportamento abrupto na absorção de água foi observado com a temperatura de $75{ }^{\circ} \mathrm{C}$. Notadamente, para esta condição, o arroz apresenta um patamar a partir das 5 horas de experimento, demonstrando o teor de água de equilíbrio dos grãos. Maskan (2002) observou comportamento similar a este estudando diferentes variedades de trigo e atribuiu esse comportamento ao fato dos grãos já terem alcançado a temperatura de gelatinização do amido.

Mudanças estruturais no arroz em casca parboilizável devido à gelatinização são constantemente observadas em

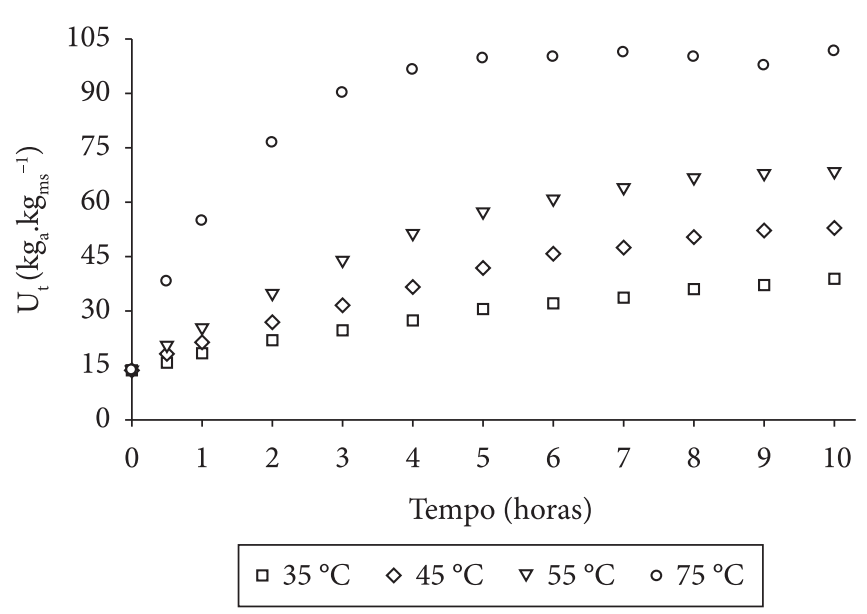

Figura 1. Valores observados para o de teor de água do arroz em casca para as temperaturas de $35,45,55$ e $75^{\circ} \mathrm{C}$. 
trabalhos dessa natureza com diferentes temperaturas de gelatinização do amido. Bakshi e Singh (1980) observaram para o arroz integral e para o arroz em casca, a temperatura de gelatinização de $85^{\circ} \mathrm{C}$; Igathithane, Chattopadhyay e Pordesimo (2005) verificaram para arroz em casca, variedade Pankaj, a temperatura de gelatinização de $72^{\circ} \mathrm{C}$, e Bello, Tolaba e Suarez (2007) observaram uma temperatura de gelatinização para o arroz integral de $60{ }^{\circ} \mathrm{C}$. Essas diferenças nas temperaturas encontradas podem ser atribuídas, principalmente às diferentes cultivares de arroz utilizadas, o que suscita a necessidade destes estudos, não só para diferentes produtos, mas também para diferentes variedades.

\subsection{Modelo de Peleg na descrição do fenômeno de absorção de água}

Para verificar a qualidade do ajuste do modelo de Peleg para representar o fenômeno da hidratação dos grãos de arroz, o modelo foi ajustado aos dados experimentais sendo determinados seus coeficientes para cada temperatura estudada.

Observa-se, na Figura 2, que o modelo se ajustou bem aos dados experimentais, o que pode ser comprovado pela correspondência entre os valores observados e estimados pelo modelo para todas as temperaturas de embebição e pelos valores do desvio padrão da estimativa $(S E)$ do erro médio relativo $(P)$ e do coeficiente de determinação $\left(\mathrm{R}^{2}\right)$ apresentados na Tabela 1 . Nesta mesma tabela, estão apresentados os coeficientes $C_{1}$ e $C_{2}$ do modelo (Equação 1) e os teores de água de equilíbrio $\left(\mathrm{U}_{\mathrm{e}}\right)^{2}$ obtidos pela Equação 2 .

Pelos resultados apresentados na Tabela 1, observa-se que o modelo mostrou-se adequado para descrever o processo de

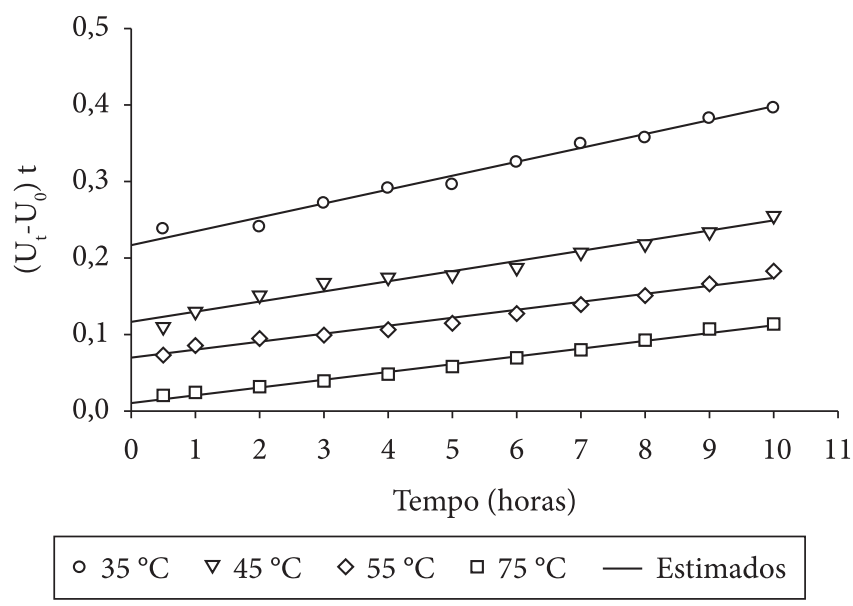

Figura 2. Valores observados e estimados pelo modelo de Peleg linearizado para as temperaturas de $35,45,55$ e $75^{\circ} \mathrm{C}$. hidratação nos grãos de arroz em casca da variedade analisada, apresentando $p$-valor para todos os coeficientes menores que 0,0001 (probabilidade da hipótese de nulidade ser verdadeira) para todas as condições, o que, segundo Resio, Aguerre e Suarez (2006), indica um bom comportamento desse modelo aos dados experimentais. Além disso, o modelo apresentou valores de $\mathrm{R}^{2}$ maiores que $97 \%$ e valores de $P$ menores que $10 \%$, o que, segundo Mohapatra e Rao (2005), representam um ajuste satisfatório do modelo aos dados experimentais. Nota-se também que os valores de "SE", assim como os valores de " $P$ ", aumentaram com o aumento da temperatura, demonstrando que o modelo melhor representou os dados experimentais para temperaturas menores (entre 35 e $55^{\circ} \mathrm{C}$ ). Cunningham, McMinn e Richardson (2007) também observaram em seu trabalho um melhor ajuste do modelo de Peleg para temperaturas mais baixas, entre 20 e $60{ }^{\circ} \mathrm{C}$, e atribuíram o fato à maior lixiviação de sólidos que ocorre nesse tipo de processo nas temperaturas mais elevadas, que não é contabilizado pelo modelo.

A constante $C_{1}$ do modelo de Peleg está relacionada com a taxa de transferência de massa, sendo que, quanto menores forem os valores de $C_{1}$ maiores serão as taxas iniciais de absorção de água (TURHAN; SAYAR; GUNASEKARAN,2002). Este coeficiente pode ser ainda relacionado com o coeficiente de difusão (CUNNINGHAM; McMINN; RICHARDSON, 2007; MASKAN, 2002). As constantes $C_{1}$ para este experimento decresceram com o aumento da temperatura, descrevendo uma relação quadrática, em função da temperatura de hidratação, conforme resultados apresentados na Tabela 2.

Resende e Corrêa (2007) e Cunningham, McMinn e Richardson (2007) também observaram que a constante $C_{1}$ decresce com a temperatura para uma faixa de 20 a $50{ }^{\circ} \mathrm{C}$ e de 20 a $80^{\circ} \mathrm{C}$, respectivamente, porém numa relação linear. Os valores encontrados de 0,20283 a 0,01138 (100 h kg $\left.{ }_{\mathrm{ms}}\right) \mathrm{kg}_{\mathrm{a}}^{-1}$ são compatíveis aos encontrados por Djomdi e Ndjouenkeu (2007), com valores variando de 0,514 a 0,007 (100 h kg $\left.{ }_{\mathrm{ms}}\right) \mathrm{kg}_{\mathrm{a}}^{-1}$, numa faixa de temperatura entre 20 e $100{ }^{\circ} \mathrm{C}$ para raízes de Cyperus esculentus.

A constante $C_{2}$ do modelo de Peleg está relacionada com a capacidade máxima de absorção de água, sendo que, quanto menor o seu valor, maior será a absorção de água do produto (RESENDE; CORRÊA, 2007). Assim como a constante $C_{1}$, a constante $C_{2}$ decresceu numa relação quadrática em relação

Tabela 2. Dependência dos coeficientes $\mathrm{C}_{1}$ e $\mathrm{C}_{2}$ do modelo de Peleg com a temperatura $(\mathrm{T})$ de hidratação.

\begin{tabular}{crcc}
\hline Coeficiente & Equação & $\mathrm{R}^{2}(\%)$ & \\
\hline $\mathrm{C}_{1}$ & $0,000129 \mathrm{~T}^{2}-0,0192 \mathrm{~T}+0,727$ & 99,38 & $(8)$ \\
$\mathrm{C}_{2}$ & $0,0000086 \mathrm{~T}^{2}-0,00114 \mathrm{~T}+0,047$ & 99,97 & $(9)$ \\
\hline
\end{tabular}

Tabela 1. Parâmetros do modelo de Peleg e teores de água de equilíbrio para hidratação do arroz em casca em função da temperatura de hidratação.

\begin{tabular}{|c|c|c|c|c|c|c|}
\hline $\mathrm{T}\left({ }^{\circ} \mathrm{C}\right)$ & $\mathrm{C}_{1}\left(100 \mathrm{~h} \mathrm{~kg}_{\mathrm{ms}} \cdot \mathrm{kg}_{\mathrm{a}}^{-1}\right)$ & $\mathrm{C}_{2}\left(100 \mathrm{~kg}_{\mathrm{ms}} \cdot \mathrm{kg}_{\mathrm{a}}^{-1}\right)$ & $\mathrm{U}_{\mathrm{e}}\left(100 \mathrm{~kg}_{\mathrm{a}} \cdot \mathrm{kg}_{\mathrm{ms}}^{-1}\right)$ & SE $\left(100 \mathrm{~kg}_{\mathrm{a}} \cdot \mathrm{kg}_{\mathrm{ms}}^{-1}\right)$ & $\mathrm{P}(\%)$ & $\mathrm{R}^{2}(\%)$ \\
\hline 35 & $0,20283^{* *}$ & $0,01985^{* *}$ & 69,4 & 0,313 & 0,91 & 98,3 \\
\hline 45 & $0,11549^{* *}$ & $0,01328^{* *}$ & 88,7 & 0,859 & 1,77 & 97,1 \\
\hline 55 & $0,06827^{* *}$ & $0,01061^{* *}$ & 108,8 & 1,514 & 2,19 & 98,0 \\
\hline 75 & $0,01138^{* *}$ & $0,01009^{* *}$ & 113,5 & 4,157 & 4,41 & 99,1 \\
\hline
\end{tabular}

p-valor $<0,0001$ (probabilidade da hipótese nula ser verdadeira). 
à temperatura de hidratação, conforme apresentado Tabela 2. Resultados semelhantes foram observados por Resende e Corrêa (2007). Os valores para essa constante variaram de 0,01985 a 0,01009 (100 kg $\mathrm{ms}) \mathrm{kg}_{\mathrm{a}}^{-1}$. Cunningham, McMinn e Richardson (2007) argumentam que a dependência de $C_{2}$ com a temperatura pode ser atribuída ao aumento do teor de água de equilíbrio do produto com o aumento da temperatura de hidratação, conforme pode ser observado neste trabalho na Tabela 2. Os valores encontrados de $C_{2}$ estão de acordo com os encontrados por Abu-Ghannam e McKenna (1997), que obtiveram valores variando de 0,0035 a 0,0107 $\left(100 \mathrm{~kg}_{\mathrm{ms}}\right) \mathrm{kg}_{\mathrm{a}}^{-1}$ para uma dada variedade de feijão.

Uma vez que as constantes $C_{1}$ e $C_{2}$ podem ser expressas em função da temperatura por meio das Equações 8 e 9, o modelo de Peleg pode ser descrito de acordo com a Equação 10:

$$
U_{t}=U_{0}+\frac{t}{\left[\begin{array}{l}
\left(1,29 \times 10^{-4} T^{2}-0,0192 T+0,727\right)+ \\
+\left(8,6 \times 10^{-6} T^{2}-0,00114 T+0,047\right)
\end{array}\right]}
$$

em que: $t$ : temperatura, ${ }^{\circ} \mathrm{C}$.

Na Figura 3, apresenta-se o gráfico de correspondência entre os valores observados experimentalmente e estimados pelo modelo de Peleg, com seus coeficientes variando em função da temperatura, para a faixa de 35 a $75^{\circ} \mathrm{C}$, bem como os valores de $P$ e $S E$.

Pela correspondência entre os valores experimentais e os estimados, bem como pela magnitude dos valores de $P$ e $S E$, nota-se que o modelo de Peleg com seus coeficientes em função da temperatura descreve adequadamente o processo de hidratação no arroz em casca.

\subsection{Coeficiente efetivo de difusão e energia de ativação}

Os valores do coeficiente efetivo de difusão para cada condição de hidratação do arroz em casca estão apresentados na Tabela 3. O coeficiente efetivo de difusão foi obtido por meio de regressão não linear da Equação 4, com aproximação de oito termos pelo método Gauss Newton, utilizando o software Statistica $6.0^{\circledR}$.

Pela Tabela 3, observa-se que os valores do coeficiente efetivo de difusão aumentaram com a elevação da temperatura, variando de $0,7987 \times 10^{-11}$ a $9,176 \times 10^{-11} \mathrm{~m}^{2} / \mathrm{s}$. Com o aumento da temperatura, a viscosidade da água diminui e, sendo esta propriedade uma medida da resistência do fluído ao escoamento, as suas variações implicam em alterações na difusão da água nos capilares do grão de modo a favorecer a sua movimentação no produto. Outro fator a que pode ser atribuído essa variação do coeficiente efetivo de difusão é que, com o aumento da

Tabela 3. Coeficiente efetivo de difusão para o arroz em casca parboilizável em função da temperatura de hidratação.

\begin{tabular}{cc}
\hline Temperatura $\left({ }^{\circ} \mathrm{C}\right)$ & Coeficiente de difusão efetivo $\times 10^{11}\left(\mathrm{~m}^{2} / \mathrm{s}\right)$ \\
\hline 35 & 0,80 \\
45 & 1,24 \\
55 & 1,76 \\
75 & 9,18 \\
\hline
\end{tabular}

temperatura, aumenta o nível de vibração das moléculas da água, contribuindo para uma difusão mais rápida.

Esse comportamento do coeficiente efetivo de difusão também foi observado por diversos pesquisadores. Addo, BartPlange e Dzisi (2006), estudando duas variedades de milho, encontraram valores variando de $7,3 \times 10^{-12}$ a $9,3 \times 10^{-12} \mathrm{~m}^{2} / \mathrm{s}$ e $6,3 \times 10^{-12}$ a $8,25 \times 10^{-12} \mathrm{~m}^{2} / \mathrm{s}$, respectivamente para as diferentes variedades, na faixa de temperatura de 30 a $60^{\circ} \mathrm{C}$, e, Bello, Tolaba e Suarez (2004), estudando três tipos de arroz, encontraram valores de 1,56 a $7,2 \times 10^{-11} \mathrm{~m}^{2} / \mathrm{s}, 2,22$ a $8,82 \times 10^{-11} \mathrm{~m}^{2} / \mathrm{s}$ e 20,5 a $47 \times 10^{-11} \mathrm{~m}^{2} / \mathrm{s}$, para o arroz em casca, descascado e polido, respectivamente na faixa de temperatura de 25 a $65^{\circ} \mathrm{C}$.

A dependência do coeficiente efetivo de difusão com a temperatura, expressa por meio da relação de Arrhenius, está apresentada na Figura 4 com a respectiva Equação de Arrhenius linearizada.

De acordo com a Figura 4, nota-se que, para o intervalo de temperatura de 35 a $55^{\circ} \mathrm{C}$, existe uma uniformidade de variação do coeficiente efetivo de difusão para esta faixa de temperatura. Todavia, uma expressiva variação desse coeficiente é observada a partir da temperatura de $55^{\circ} \mathrm{C}$ (ponto de quebra). Bakshi e Singh (1980) também observaram esse comportamento para a representação de Arrhenius e atribuíram esse ponto de quebra

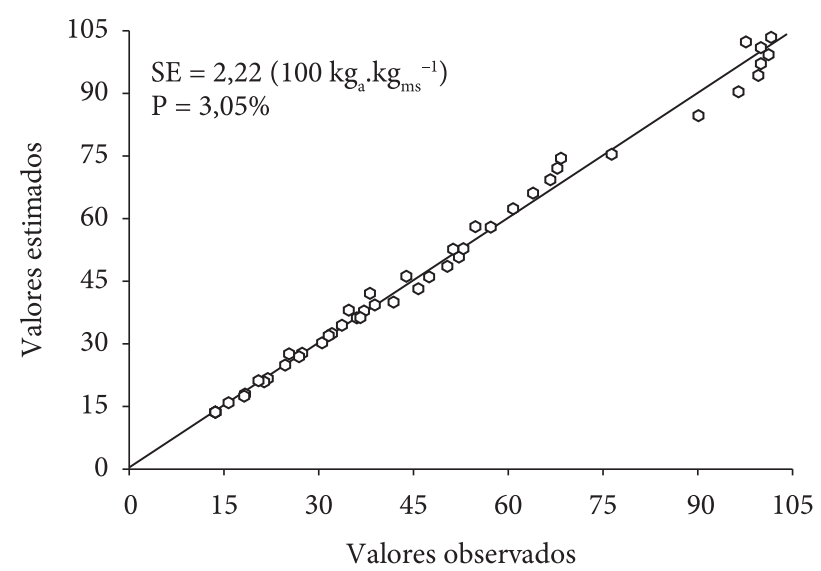

Figura 3. Correspondência entre os valores experimentais e os estimados pelo modelo de Peleg com seus coeficientes variando em função da temperatura de hidratação.

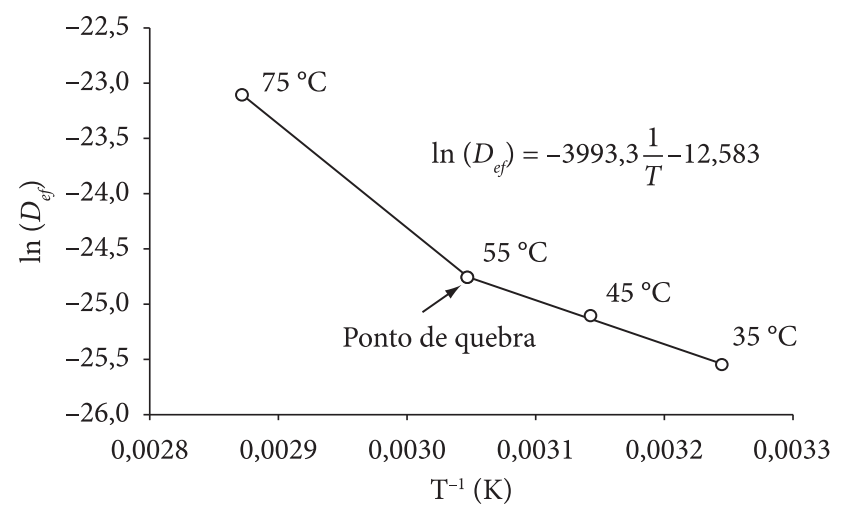

Figura 4. Representação de Arrhenius para o coeficiente efetivo de difusão para o arroz em casca parboilizável. 
à temperatura na qual ocorre a total gelatinização do amido. Entretanto, não se pode afirmar a temperatura na qual ocorre completa gelatinização do amido para este trabalho, devido ao expressivo intervalo de variação de temperatura. No entanto, pode se afirmar que a temperatura na qual esse fenômeno acontece está entre as temperaturas de 55 e $75^{\circ} \mathrm{C}$.

Assim, para a determinação da energia de ativação da difusão de água no arroz, durante o processo de hidratação, considerou-se a faixa de temperatura de 35 a $55^{\circ} \mathrm{C}$, e a Equação de Arrhenius linearizada referente à essa faixa de temperatura esta apresentada abaixo (Equação 11):

$\ln \left(D_{e f}\right)=-\frac{32,2}{R T_{a}}-12,583$

Nota-se pela Equação 11 que o valor da energia de ativação foi de $32,3 \mathrm{~kJ} \cdot \mathrm{mol}^{-1}$. O valor encontrado está de acordo com o obtido por Resio, Aguerre e Suarez (2003) com uma energia de ativação de $32,1 \mathrm{~kJ} \cdot \mathrm{mol}^{-1}$ para a difusão de água em sementes de amaranto submetidas ao processo de hidratação às temperaturas entre 30 e $64^{\circ} \mathrm{C}$.

\section{Conclusões}

De acordo com os resultados encontrados no presente trabalho, foi possível concluir que a hidratação do arroz da variedade IRGA 242 pode ser descrita satisfatoriamente pelo modelo de Peleg; as constantes $C_{1}$ e $C_{2}$ do modelo de Peleg decresceram quadraticamente com o aumento da temperatura de hidratação; a taxa de hidratação aumenta com a elevação da temperatura, com o coeficiente efetivo de difusão variando de 0,80 a $9,18 \times 10^{-11} \mathrm{~m}^{2} / \mathrm{s}$ para a faixa de temperatura de 35 a $75^{\circ} \mathrm{C}$; a energia de ativação para o processo que relacionou o coeficiente efetivo de difusão com a temperatura foi de $33,2 \mathrm{~kJ} \cdot \mathrm{mol}^{-1}$.

\section{Referências bibliográficas}

ABU-GHANNAM, N.; McKENNA, B. The Application of Peleg's Equation to Model Water Absorption During the Soaking of Red Kidney Beans (Phaseolus vulgaris L.). Journal of Food Engineering, v. 32, p. 391-401, 1997.

ADDO, A.; BART-PLANGE, A.; DZISI, K. Water Absorption characteristics of Obatanpa and Mamaba Maize Hybrids (Zea mays). International Journal of Food Engineering, v. 2, n. 3, p. 7, 2006.

BAKSHI, A. S.; SINGH, R. P. Kinetics of water diffusion and starch gelatinization during rice parboiling. Journal of Food Science, V. 45, n. 5, p. 1387-1392, 1980.

BAYRAM, M.; KAYA, A.; ÖNER, M. D. Changes in properties of soaking water during production of soy-bulgur. Journal of Food Engineering, v. 61, p. 221-230, 2004.

BELLO, M.; TOLABA, M. P.; SUAREZ, C. Factors affecting water uptake of rice grain during soaking. Lebensmittel-Wissenschaft und -Technologie, v. 37, p. 811-816, 2004.

BELLO, M.; TOLABA, M. P.; SUAREZ, C. Water absorption and starch gelatinization in whole rice grain during soaking. LebensmittelWissenschaft und -Technologie, v. 40, p. 313-318, 2007.

BRASIL. Ministério da Agricultura e Reforma Agrária. Secretaria Nacional de Defesa Agropecuária. Regras para análises de sementes. Brasília, DF, 1992. 365 p.

BROOKER, D. B.; BAKKER-ARKEMA, F. W.; HALL, C. W. Drying and storage of grains and oilseeds. Westport: The AVI Publishing Company, 1992.
CUNNINGHAM, S. E.; MCMINN, W. A. M.; RICHARDSON, P. S. Modelling water absorption of pasta during soaking. Journal of Food Engineering, v. 82, p. 600-607, 2007.

DJOMDI, R. E.; NDJOUENKEU, R. Soaking behaviour and milky extraction performance of tiger nut (Cyperus esculentus) tubers. Journal of Food Engineering, v. 78, p. 546-550, 2007.

GARCÍA-PASCUAL, P. et al. Morchella esculenta (morel) rehydration process modelling. Journal of Food Engineering, v. 72, p. 346-353, 2006.

GELY, M. C.; GINER, S. A. Diffusion Coefficient Relationships during drying of Soya Bean Cultivars. Biosystems Engineering, v. 96, p. 213-222, 2007.

GELY, M. C.; SANTALLA, E. M. Moisture diffusivity in quinoa (Chenopodium quinoa Willd.) seeds: Effect of air temperature and initial moisture content of seeds. Journal of Food Engineering, v. 78, p. 1029-1033, 2007.

GOWEN, A. et al. Modelling the water absorption process in chickpeas (Cicer arietinum L.) - The effect of blanching pre-treatment on water intake and texture kinetics. Journal of Food Engineering, v. 78, p. 810-819, 2007.

IGATHINATHANE, C.; CHATTOPADHYAY, P. K.; PORDESIMO L. O. Combination soaking procedure for rough rice parboiling. Transactions of the ASAE, v. 48, n. 2, p. 665-671, 2005.

INSTITUTO BRASILEIRO DE GEOGRAFIA E ESTATÍSTICA IBGE. Levantamento Sistemático da Produção Agrícola. [S.l.], 2007. Disponível em: <http://www.ibge.gov.br/home/estatistica/ indicadores/agropecuaria/lspa/lspa_200709_5.shtm>. Acesso em: 08 nov. 2007.

KORNARZYNSKI, K.; PIETRUSZEWSKI, S.; LACEK, R. Measurement of the water absorption rate in wheat grain. International Agrophysics, v. 16, p. 33-36, 2002.

LUCAS, T.; RAY, D. L.; MARIETTE, F. Kinetics of water absorption and solute leaching during soaking of breakfast cereals. Journal of Food Engineering, v. 80, p. 377-384, 2007.

MASKAN, M. Effect of maturation and processing on water uptake characteristics of wheat. Journal of Food Engineering, v. 47, p. 51-57, 2001.

MASKAN, M. Effect of processing on hydration kinetics of three wheat products of the same variety. Journal of Food Engineering, v. 52, p. 337-341, 2002.

MOHAPATRA, D.; RAO, P. S. A thin layer drying model of parboiled wheat. Journal of Food Engineering, v. 66, p. 13-18, 2005.

PELEG, M. An empirical model for the description of moisture sorption curves. Journal of Food Science, v. 52, n. 4, p. 1216-1219, 1988.

RESENDE, O.; CORRÊA, P. C. Modelagem matemática do processo de hidratação de sementes de feijão, Acta Scientiarum Agronomy, v. 29, n. 3, p. 373-378, 2007.

RESIO, A. C.; AGUERRE, R. J.; SUAREZ, C. Hydration kinetics of amaranth grain. Journal of Food Engineering, v. 72, p. 247-253, 2006.

RESIO, A. C.; AGUERRE, R. J.; SUAREZ, C. Study of some factors affecting water absorption by amaranth grain during soaking. Journal of Food Engineering, v. 60, p. 391-396, 2003.

ROCA, E. et al. Effective moisture diffusivity modeling food structure and hygroscopicity. Food Chemistry, v. 106, p. 1428-1437, 2008.

SAYAR, S.; TURHAN, M.; GUNASEKARAN, S. Analysis of chickpea soaking by simultaneous water transfer and water-starch reaction. Journal of Food Engineering, v. 50, p. 91-98, 2001.

SEYHAN-GÜRTAS, F.; MEHMET, A. K. M.; EVRANUZ, E. O. Water Diffusion Coefficients of Selected Legumes Grown in Turkey As Affected by Temperature and Variety. Journal of Agriculture and Forestry, v. 25, p. 297-304, 2001.

TURHAN, M.; SAYAR, S.; GUNASEKARAN, S. Application of Peleg model to study water absorption in chickpea during soaking. Journal of Food Engineering, v. 53, p. 153-159, 2002. 